\title{
Investigation of Plasma Formation with ns Laser by Using Focused Sub-ns Laser Probe
}

\author{
Shunsuke Ikeda*, Takeshi Kanesue, and Masahiro Okamura \\ Collider-Accelerator Department, Brookhaven National Laboratory, NY, USA
}

\begin{abstract}
A laser ablation plasma formation process was investigated by shooting another laser beam. The initial plasma was created by a mildly focused nanosecond laser beam and the probe laser, which has about ten times shorter pulse length with tightly focussed condition, was irradiated on it. By analysing high temperature plasma created by the probe laser, we could detect an interaction between the initial low temperature plasma and the probe laser. The interaction caused that the velocity of high temperature plasma generated by the sub-ns laser became smaller and the amount of the highly charged ions decreased. We found that the interaction does not occur during the irradiation of the first half of the ns laser. This fact indicates that the plasma is not produced during the first half of the ns laser.
\end{abstract}

\section{Introduction}

Laser ablation plasma is suitable for providing intense ion flux with various charge states ranging from singly charged to fully stripped. The advantage of the ion production scheme is that a large number of ions can be produced from most of the solid materials under high vacuum condition. The induced ions can be applied to many fields such as an ion source for particle accelerators[1].

For production of low charge state ions, laser intensity on a target surface is close to the laser ablation threshold. Typically, the threshold is $10^{8} \mathrm{~W} / \mathrm{cm}^{2} \sim 10^{9} \mathrm{~W} / \mathrm{cm}^{2}$ for nanosecond and sub-nanosecond lasers [2-4]. Most of the ions are singly charged for the laser intensity $[5,6]$. In the intensity region, the plasma is not uniform but evolves temporally and spatially. To investigate the plasma formation, we are proposing to use a focused another laser pulse with a shorter pulse width as a probe. If one irradiates a laser target with a focused subnanosecond laser during the irradiation of a mildly focused ns laser pulse, the interaction of the sub-ns laser and the ns-laser plasma depends on the property of the ns-laser plasma. The interaction makes the difference of the plasma property after the expansion. Therefore, one can investigate the property of the ns-laser plasma with temporal and spatial resolutions by analysing the plasma after the expansion with different spot positions and timings of the sub-ns laser.

As a first step of the investigation, we measured ion current and charge state distribution of plasma with different timings of the sub-ns laser focused on the center of the ns laser spot. Based on the results, we discussed the production process of the ns-laser plasma.

\section{Experimental setup}

Figure 1 shows the experimental setup. An aluminium plate was irradiated with a nanosecond and a subnanosecond lasers. The target plate was placed on linear stages and moved every laser shot. The target system was installed in the grounded chamber evacuated to less than $4 \times 10^{-4} \mathrm{~Pa}$.

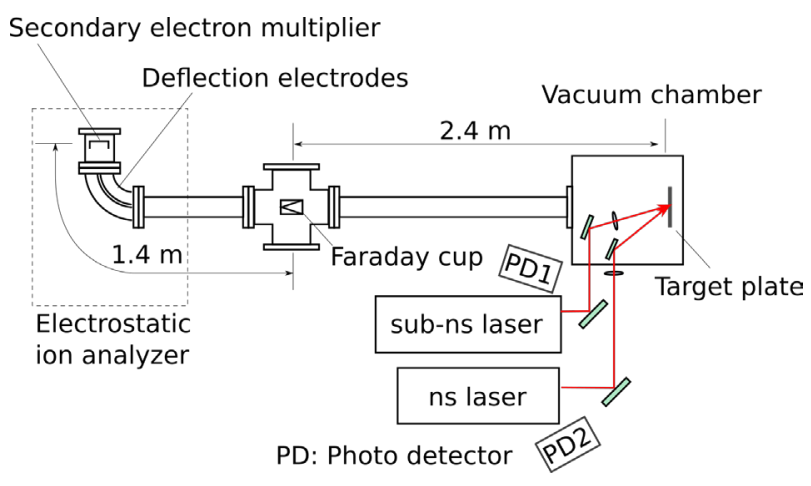

Fig. 1. Experimental setup.

The nanosecond laser pulse was generated with a Nd:Yag laser (THALES SAGA 230). The pulse duration was $6 \mathrm{~ns}$ and the wave length was $1064 \mathrm{~nm}$. The energy, the area, and the intensity of the laser pulse on the target were $750 \mathrm{~mJ}, 0.16 \mathrm{~cm}^{2}$, and $8 \times 10^{8} \mathrm{~W} / \mathrm{cm}^{2}$, respectively. The incident angle was 40 degree with respect to the target normal. The sub-nanosecond laser pulse was

\footnotetext{
Corresponding author: SIkeda@bnl.gov
} 
generated with a Nd:Yag laser (Ekspla SL230). The pulse duration, the wave length, and the energy on the target were $170 \mathrm{ps}, 1064 \mathrm{~nm}$, and $300 \mathrm{~mJ}$, respectively. The laser pulse focused by a plano-convex lens with the focal length of $120 \mathrm{~mm}$. The distance between the target and the lens was adjusted to obtain a minimum time of flight of the plasma traveling from the target to a Faraday cup. The laser intensity was less than $1 \times 10^{14}$ $\mathrm{W} / \mathrm{cm}^{2}$ that was estimated by assuming a Gaussian beam. The incident angle was 20 degree with respect to the target normal.

During the experiment, we watched the laser pulses near the lasers with photo detectors, PD1 and PD2. The ns and sub-ns laser pulses arrived at the target with an interval time as illustrated in Fig. 2. We define interval time, $d t=$ (arrival time of sub-ns laser peak) - (arrival time of ns laser peak). For $d t=0$, the peaks of the nslaser pulse and the sub-ns laser pulse arrived simultaneously. For $d t>0$ (or $<0$ ), the sub-ns laser arrived after (or before) the ns laser.

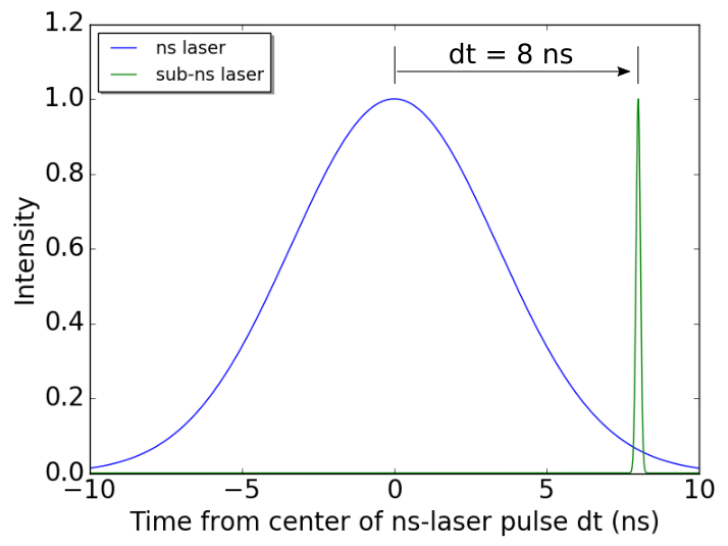

Fig. 2. Concept of time sequence of two laser pulses to explain the definition of interval time between two lasers, $d t$. The figure shows the case for $d t=8 \mathrm{~ns}$.

A current detector at $2.4 \mathrm{~m}$ far from the target was used to measure the plasma ion current. It was composed of a grounded aperture with the diameter of $10 \mathrm{~mm}$, negatively biased metal mesh, and Faraday cup. The mesh bias was determined to be $-5 \mathrm{kV}$ to obtain saturated ion current.

The charge state distribution was measured with an electrostatic ion analyser [5, 7]. During the charge state analysis, the Faraday cup was retracted.

\section{Results and discussion}

Figure 3 shows the plasma ion current waveforms. The horizontal axis is the time from the laser irradiation. The red, blue, and green curves correspond with the waveforms with the sub-ns laser, ns laser, and both lasers with a certain interval time, respectively. Because laser ablation plasma expands rapidly after the production, one can assume that the ions travel without collisions to the detector far from the target compared with the laser spot [8]. Under the assumption, the velocities of the ions are proportional to the inverses of the time of flights. The figure shows that the ns laser generated larger and slower plasma while the sub-ns laser generated smaller and faster plasma. When both lasers were used, the current waveform had two peaks. The time at the faster and slower peaks were around the waveforms with the sub-ns and ns lasers. This shows that the faster and slower peaks were generated mainly by the sub-ns and ns lasers, respectively.

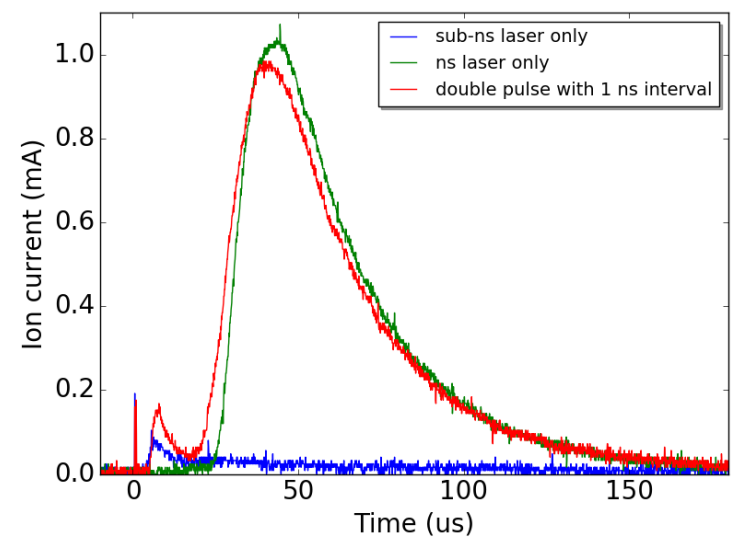

Fig. 3. Plasma ion current waveforms measured by a Faraday cup with sub-ns laser (blue), ns laser (green), and both lasers (red).

To investigate the influence of the time interval on the faster plasma, we measured plasma ion current with different the time intervals, $d t$. Figure 4 displays the wave forms around $10 \mu \mathrm{s}$ for the time intervals $d t=-1 \mathrm{~ns}$ (blue), $0 \mathrm{~ns}$ (green), and $8 \mathrm{~ns}$ (red). For comparison, the waveform only with the sub-ns laser is also shown (black). For $d t<-1 \mathrm{~ns}$, the ion current did not change as shown in the similarity between the waveforms only with the sub-ns laser and with $d t=-1$ ns interval. For larger $d t$, the height of the current peak became larger and the peak time also became larger. 


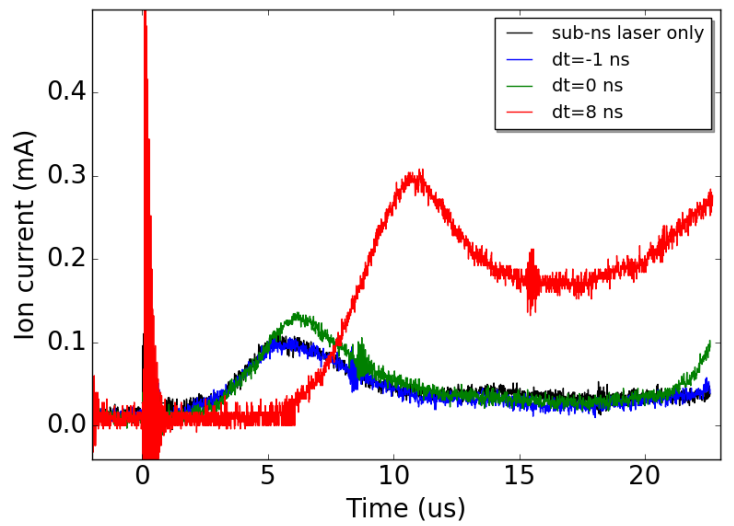

Fig. 4. Plasma ion current waveforms measured around $10 \mu \mathrm{s}$ for the time intervals $\mathrm{dt}=-1 \mathrm{~ns}$ (blue), $0 \mathrm{~ns}$ (green), and $8 \mathrm{~ns}$ (red). For comparison, the waveform only with the sub-ns laser is also shown (black), which is overlapped with the blue curve.

Figure 5 shows the veocity estimated from the peak time and the height of the peak current as a function of $d t$. The graph shows clearly that the peak velocity decreased and the peak current increased monotonically with the increase of $\mathrm{dt}$ for $d t>-1 \mathrm{~ns}$.

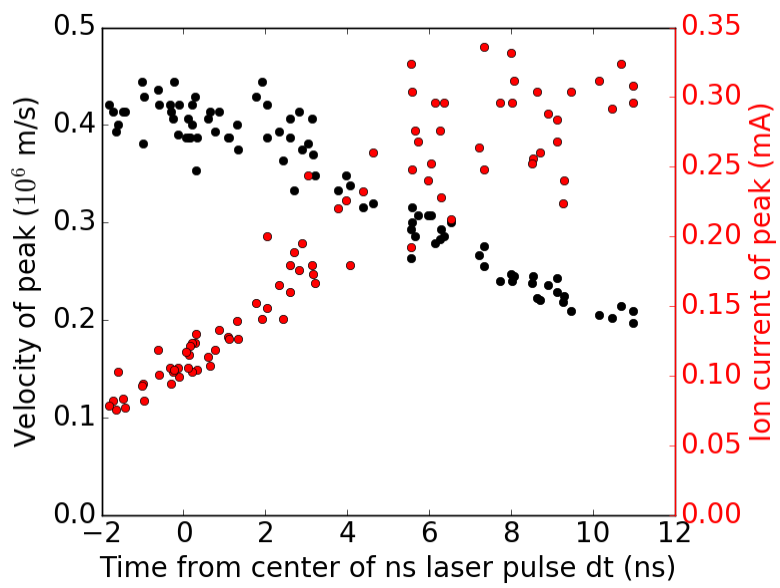

Fig. 5. Velocity estimated from the peak time (black) and height of the peak ion current (red) as a function of $d t$.

Figures 6(a) and 6(b) show the time structures of (a) $\mathrm{Al}^{11+}$ and (b) $\mathrm{Al}^{8+}$ generated only with the sub-ns laser (black) and the both laser pulses for $d t=8 \mathrm{~ns}$ (red). The horizontal axis is the time at the Faraday cup estimated by assuming the time of flight is proportional to the traveling distance of the ions. The vertical axis is the voltage of the secondary electron multiplier used in the electrostatic ion analyser. Although calibrations are needed to compare the voltage among different species, one can compare the voltages for the same species directly. As shown in the figures and the similar figures for the other charge states that are not shown here, it was found that the amounts of the highly charged ions $(12+\sim$ $9+)$ and the ions with lower charge states $(<8+)$ decreased and increased, respectively, when the both pulsed laser was used. The decrease of the highly charged ions around $5 \mu \mathrm{s}$ and the increase of the lower charge states for the measurement time later than $10 \mu \mathrm{s}$ are consistent with the difference between the ion current waveforms shown in Fig.4.

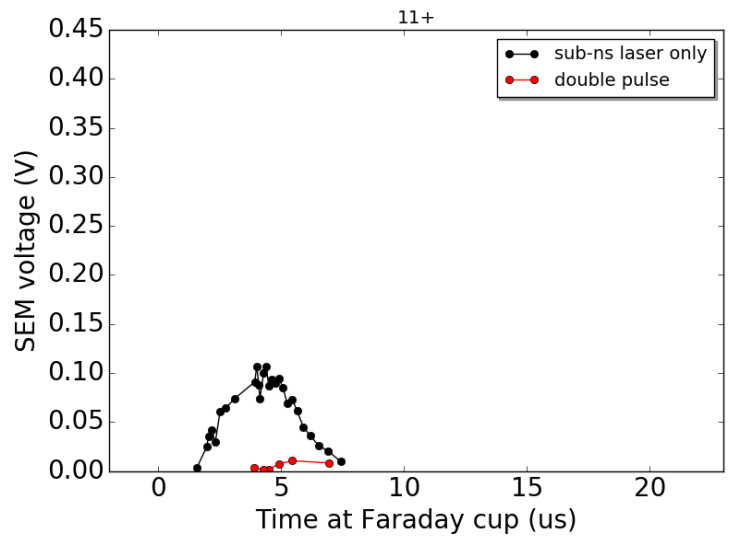

(a)

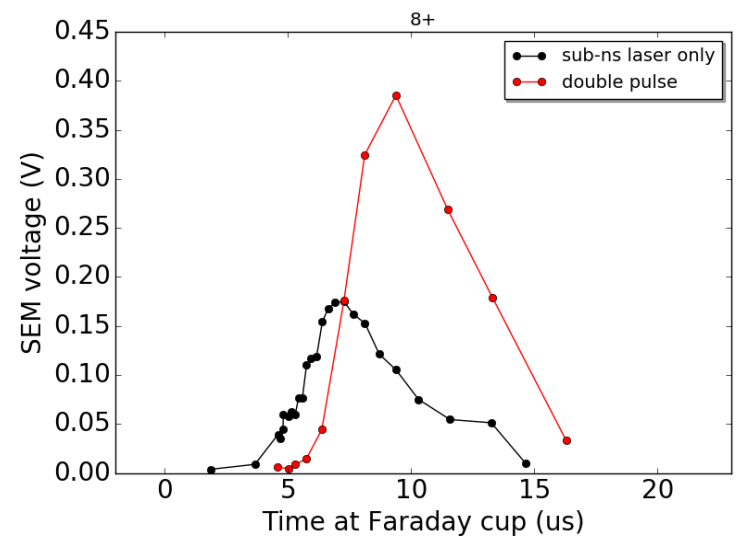

(b)

Fig. 6. Time structures of (a) $\mathrm{Al}^{11+}$ and (b) $\mathrm{Al}^{8+}$ generated only with the sub-ns laser (black) and the both laser pulses for $d t=8$ ns (red) as a function of time at the Faraday cup.

We observed that the velocity of the faster plasma decreased and the highly charged ions decreased while the ions with lower charge states increased when the both laser pulses were irradiated. The changes indicate that the plasma temperature was lower. This shows the influence of the ns-laser plasma. By the first ns laser, large volume of the plasma was generated. The volume was larger than the plasma generated only with the subns laser as guessed from the larger plasma ion current shown in Fig. 3. Then, the energy of the sub-ns laser was distributed to the larger ns-laser plasma, resulting in the lower energy per ion and temperature. 
The influence of the ns-laser plasma was observed only for $d t>-1 \mathrm{~ns}$. This shows that the ns-laser plasma dense enough to interact with the second sub-ns laser pulse was produced after the first half of the ns laser pulse. In other word, the plasma was not produced before the first half of the laser pulse.

During this experiment, the focused sub-ns laser was irradiated on the center of the ns laser spot. If one scans the sub-ns within the ns laser spot, one can obtain the spatial information about the plasma production.

\section{Conclusions}

We investigated the evolution of the plasma generated with mildly focused ns laser by using sub-ns laser focused on a target within the ns laser spot. We measured the plasma ion current with a Faraday cup with the different interval time between the ns and sub-ns lasers. Then we measured the charge state distribution with an electrostatic ion analyser. We observed that the velocity of fast plasma generated mainly by the sub-ns laser became smaller and the amount of the highly charged ions decreased. This resulted from the interaction between the ns-laser plasma and the sub-ns laser. We found that the interaction did not occur during the irradiation of the first half of the ns laser. This fact indicates that the plasma is not produced during the first half of the ns laser.

This work was supported by the US Department of Energy under contract number DE-SC0012704.

\section{References}

1. T. Kanesue, et al., "The Commissioning of the Laser Ion Source For RHIC EBIS", in proceedings of IPAC2014, Dresden, Germany, 2014, 1890-1892.

2. S. Amoruso, et al., J. Phys. B-At. Mol. Opt., 32(14), R131 (1999).

3. R. B. Stuart, et al., J. Opt. Soc. Am. B, 13(2), 459 (1996).

4. R. B. Stuart, et al., Phys. Rev. B, 53(4), 1749 (1996)

5. S. Ikeda, et al., Rev. Sci. Instrum. 85(2), 02 B913 (2014).

6. T. Kanesue, et al., "Measurement of Ion Beam from Laser Ion Source for RHIC EBIS", in proceedings of EPAC2008, Genoa, Italy, 2008, 421-423.

7. E, Woryna, et al., Laser Part Beams, 14(3), 293 (1996).

8. R. Kelly and R. Dreyfus, Surf. Sci., 198(1), 263 (1998) 